\title{
Numerical Simulation of Fatigue Crack Initiation in Thin-walled High Strength Steel as Modeled by Voronoi-polygons
}

\author{
Shunsuke TOYODA, ${ }^{1)}$ Hideto KIMURA, ${ }^{1)}$ Yoshikazu KAWABATA, ${ }^{2)}$ Shota HASHIMOTO, ${ }^{3)}$ \\ Naotake YOSHIHARA ${ }^{3)}$ and Jun'ichi SAKAI ${ }^{4)}$ \\ 1) Steel Research Laboratory, JFE Steel Corporation, 1-1 Kawasaki-cho, Handa, Aichi 475-8611 Japan. \\ 2) Chita Works, JFE Steel Corporation, 1-1 Kawasaki-cho, Handa, Aichi 475-8611 Japan. \\ 3) JFE Techno-Research Corporation, 1-1 Minamiwatarida-cho, Kawasaki-ku, Kawasaki, Kanagawa 210-0855 Japan. \\ 4) Faculty of Science and Engineering, Waseda University, 3-4-1 Okubo, Shinjuku-ku, Tokyo 169-8555 Japan.
}

(Received on May 26, 2010; accepted on July 22, 2010)

\begin{abstract}
The fatigue endurance of TS $590 \mathrm{MPa}$ grade low-alloy precipitation strengthened steel was numerically and experimentally examined. The microstructure was modeled using two-dimensional Voronoi polygons. Heterogeneous stress distributions were calculated using the finite element method, taking elastic anisotropy into consideration. The number of cycles before crack initiation was estimated based on the Tanaka-Mura model. By taking into account the effects of the cyclic strain of the preceding cracks, a definable macroscopic crack initiation cycle was obtained. An actual tensile and compression fatigue test was conducted on the same steel. The stress amplitude decreased as the cycle number increased. Distinct dislocation cell structure was not observed by TEM analysis. The experimental strain fatigue limit was, to some extent, lower than that of the simulation. Surface effects, specimen homogeneity, selection of slip system, and dislocation reversibility are mentioned as the probable causes for the difference.
\end{abstract}

KEY WORDS: crack initiation; fatigue life; cyclic deformation; simulation; high strength steel; microstructure; precipitation hardening.

\section{Introduction}

The fatigue-life curve of thin-walled, high strength steel is one of the most important properties for automotive structural parts. ${ }^{1-10)}$ Significant attention has been devoted to this area for many years. However, prediction of fatiguelife still remains a challenging research subject. One of the difficulties of fatigue-life prediction is that the whole fatigue process consists of three consecutive stages ${ }^{11)}$ : (1) fatigue hardening and/or softening, (2) micro-crack nucleation, and (3) crack propagation ending in final fracture. Therefore, to set up a versatile prediction model, each stage must be formulated individually.

Tanaka and Mura $^{12)}$ proposed an initiation predicting model that assumes the micro-cracks are initiated by irreversible dislocation pile-ups in slip bands as expressed by the following equation:

$$
N i=\frac{8 G W s}{\pi(1-v) d(\Delta \tau-2 k)^{2}}
$$

where $N i$ denotes the number of cycles to crack initiation in the specific slip band, $G$ is the shear modulus, $W_{S}$ is the fracture energy per unit area, $v$ is Poisson's ratio, $d$ is the length of the slip band, $\Delta \tau$ is the resolved shear stress range on the slip plane, and $k$ is the friction force of a dislocation on the slip plane. $\mathrm{Ni}$ corresponds to fatigue hardening and/or softening during the micro-crack nucleation stages.
Brückner-Foit and Huang ${ }^{13)}$ estimated the number of cycles before crack initiation in martensite stainless steel on the basis of the Tanaka-Mura model. The simulation model yields a relationship between crack densities and the number of load cycles. The results describe the generation of discrete crack embryo well. However, the transition point from a microscopic to a macroscopic crack ( $\mathrm{Ni}$-macro) is not clearly defined yet.

In this study, the crack initiation of TS $590 \mathrm{MPa}$ grade low-alloy steel was numerically simulated based on Eq. (1). By taking into account the effects of the cyclic strain of the preceding cracks, the definable macroscopic crack initiation cycle is obtained. At the same time, an actual tensile and compression fatigue test was conducted on the same steel. Then, the dependence of fatigue-life on strain amplitude is discussed based on the simulation and experimental results.

\section{Experimental}

\subsection{Numerical Simulation of Fatigue Crack Initiation}

Crack initiation was numerically simulated by elastoplastic analysis using the general-purpose finite element method code ABAQUS, based on Eq. (1). This equation, derived from a dislocation pileup model, gives a $\mathrm{Ni}$ for each grain. A two-dimensional analytical square area of $500 \times$ $500 \mu \mathrm{m}$ that included about one hundred grains was modeled using the Voronoi tessellation method as shown in Fig. 
1. The cracking slip plane was assumed to be $\{011\}$. The slip direction was inclined by $45^{\circ}$ with respect to the principal axis of each grain's coordinate system. The potential crack path, which is also shown in Fig. 1, was defined as the slip plane passing through the gravity center of the each grain. The average slip plane length, $d$, was $52 \mu \mathrm{m}$. Figure 2 shows the finite element mesh of the random grain structure containing potential crack paths. The steel was assumed to have a yield strength of $562 \mathrm{MPa}$, a tensile strength of $644 \mathrm{MPa}$, elongation to fracture of $28 \%$, and uniform elongation of $14 \%$. The stress was calculated on the basis that the steel was anisotropic in the elastic range and isotropic in the plastic range. The elastic constants were $\mathrm{C} 11=\mathrm{C} 22=\mathrm{C} 33=233 \mathrm{GPa}, \quad \mathrm{C} 12=\mathrm{C} 13=\mathrm{C} 23=135$ $\mathrm{GPa}$, and $\mathrm{C} 44=\mathrm{C} 55=\mathrm{C} 66=118 \mathrm{GPa}$. The stress - -strain curve was approximated by Eq. (2) for the elastic range and by Eq. (3), the Ludwik expression, for the plastic range.

$$
\begin{gathered}
\sigma(\mathrm{MPa})=211000 \times \varepsilon \quad(\varepsilon \leq 0.00266) \ldots \ldots \ldots \\
\sigma(\mathrm{MPa})=1358 \times(0.391+\varepsilon)^{0.945} \quad(\varepsilon>0.00266) \ldots
\end{gathered}
$$

where $\sigma$ is the flow stress and $\varepsilon$ is the strain.

Von Mises yield criterion was used. The stress distribution was calculated using the strain amplitude control mode. The strain amplitude $\Delta \varepsilon / 2=0.266 \% \quad(=0.00266)$ corresponds to the yield stress of the steel. The $N i$ of each

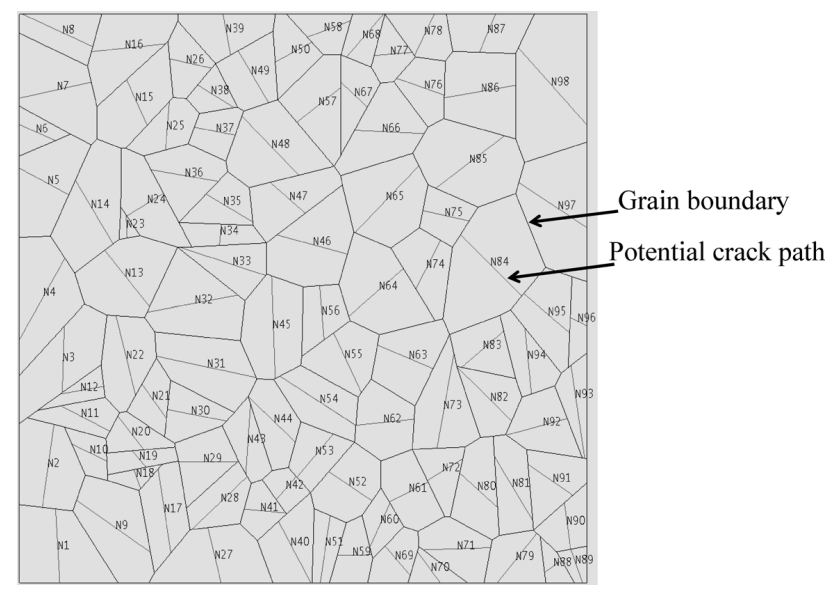

Fig. 1. Analytical area modeled by Voronoi tessellation.

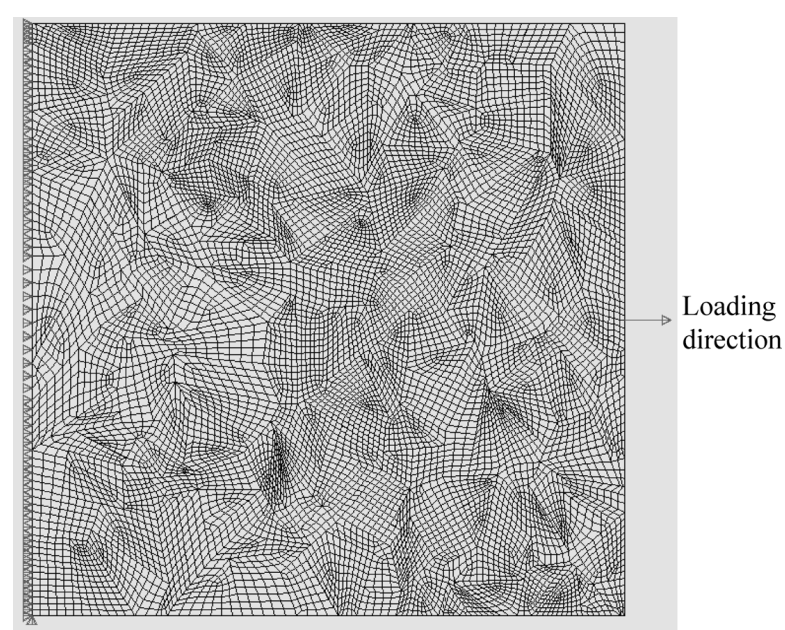

Fig. 2. Finite element mesh of random grain structure containing potential crack paths. grain was calculated by Eq. (1) following the stress distribution analysis. The material parameters were presumed as $W s=2.0 \mathrm{~kJ} / \mathrm{m}^{2}$ and $k=108 \mathrm{MPa}$. These values were reported by Hoshide et al. for S35C grade carbon steel. ${ }^{14)}$ The grain that had the minimum $N i$ was manually cracked at the location of its potential crack path. Then, the stress distribution was recalculated considering the local stress concentration caused by the grain cracking. The calculation was repeated until eight grains were cracked, which corresponds to about $12 \%$ of the total grains. The grains that had contact with the analytical boundary were eliminated from cracking manipulation.

\subsection{Strain Controlled Tension and Compression Fa- tigue Test}

The steel was hot-rolled in a laboratory mill and had the same tensile properties described above. The chemical composition of the steel used was $0.09 \% \mathrm{C}, 0.2 \% \mathrm{Si}, 1.6 \%$ $\mathrm{Mn}, 0.07 \% \mathrm{Ti}, 0.03 \% \mathrm{Nb}$, and $0.1 \% \mathrm{Cr}$. Figure 3 shows the hot-rolling conditions. Laboratory vacuum-fused and hot-rolled $50 \mathrm{~mm}$ thick plates were hot-rolled into $4 \mathrm{~mm}$ thick sheets after reheating at $1250^{\circ} \mathrm{C}$ for $1 \mathrm{~h}$. The finishing temperature was controlled at $850^{\circ} \mathrm{C}$, and the sheets were furnace-cooled to room temperature after keeping them at $575^{\circ} \mathrm{C}$ for $1 \mathrm{~h}$. The tensile properties of the specimens were the same as described in the previous section. Figure 4 shows the optical micrograph of the steel. The average ferrite grain size, $d_{f}$, was $8.4 \mu \mathrm{m}$. Figure 5 shows the fatigue test specimen with a $2 \mathrm{~mm}$ thickness and an $8 \mathrm{~mm}$ long parallel portion. The surface of the specimen was finished with

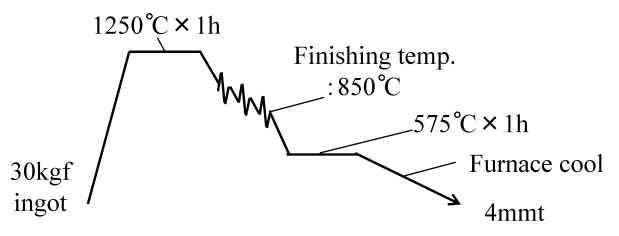

Fig. 3. Hot-rolling condition of the specimen.

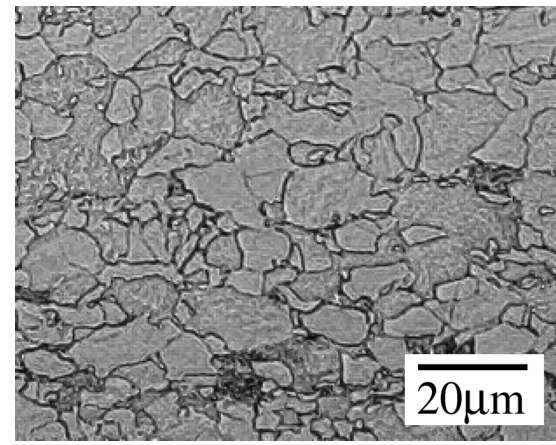

Fig. 4. Optical micrograph of the steel.

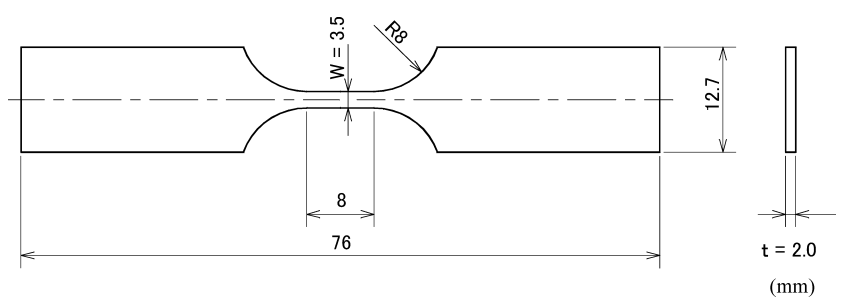

Fig. 5. Fatigue test specimen. 

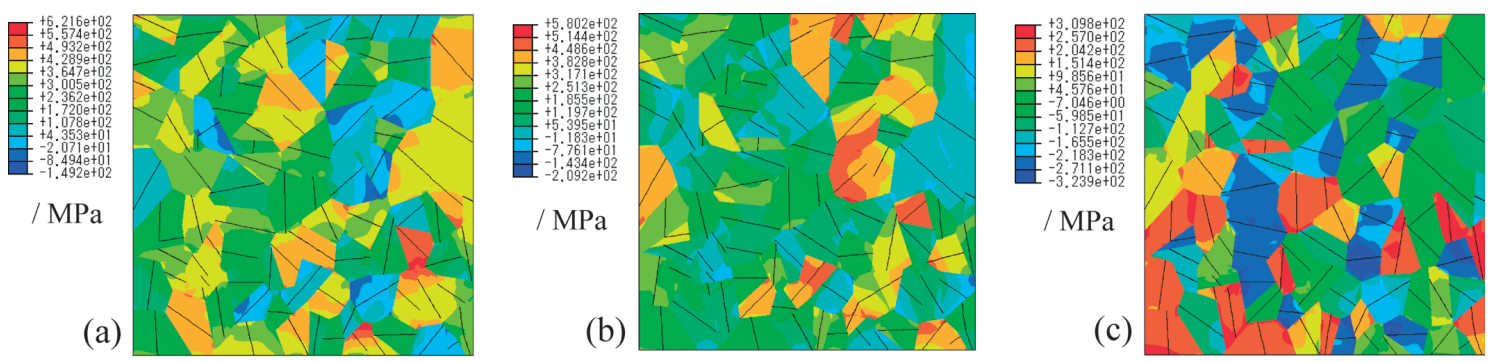

Loading direction

Fig. 6. Distribution of each stress component expressed by its local coordinate system: (a) horizontal direction stress, (b) vertical direction stress, (c) shear stress.

\#800 wet abrasive paper. A tensile and compression fatigue test was conducted under a strain ratio of $R=-1$, a repetition rate of $0.5 \mathrm{~Hz}$, and a waveform that was triangular. Strain amplitude, $\Delta \varepsilon / 2$, was varied from 0.21 to $0.27 \%$, which is around the yield stress level of the steel. The strain amplitude was gradually increased during the first ten cycles from an amplitude that was $0.03 \%$ less to the aiming amplitude. The strain-stress hysteresis curve was recorded in $1-10,20,50,70,100,200,500,700,1000 \ldots$ cycles until end of the test. When the specimen ruptured or the stress decreased by $25 \%$ from its maximum, the fatigue test was finished.

Scanning electron microscope (SEM) observation was conducted on the fracture surface and the cross-section of the specimen after the fatigue test. Transmission electron microscope (TEM) observation of the dislocation structure was carried out for some samples after the fatigue test.

\section{Results}

\subsection{Numerical Simulation of Fatigue Crack Initiation}

Figure 6 shows the distribution of each stress component as expressed by a local coordinate system. The effect of crystallographic anisotropy in each grain on stress distribution is observed. Figure 7 shows the Mises stress distribution when one crack is generated. The figure in the parenthesis is the ordered number of $N i$ in ascending sequence. This order represents the latent cracking potential. The latent cracks are distributed randomly. Figure 8 shows the Mises stress distribution when eight cracks are generated. Cracks tend to be clustered due to the local stress concentration induced by preexisting cracks. Local stress concentration can be seen on the fringe of the cracks.

Figure 9 shows the relationship between crack density and cycle number. When the effects of the cyclic strain of the preceding cracks were taken into account by Miner's rule, ${ }^{15)}$ the crack density increased more rapidly. In addition, an abrupt increase of crack density took place after several cracks were initiated. This definable abrupt increase in crack density can be considered a macroscopic crack initiation.

\subsection{Strain Controlled Tension and Compression Fa- tigue Test}

Figure 10 shows the relationship between stress amplitude and cycle number. The stress amplitude of the first ten cycles was corrected by an amount corresponding to the

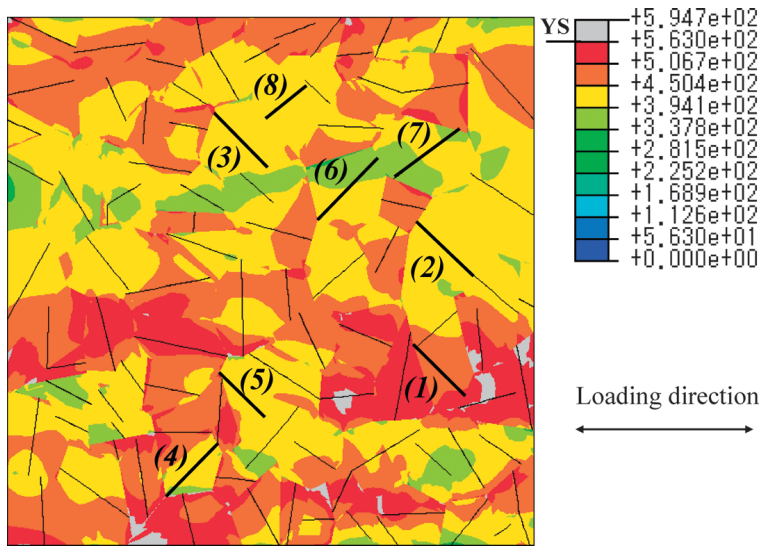

Fig. 7. Mises stress distribution when one crack is generated.
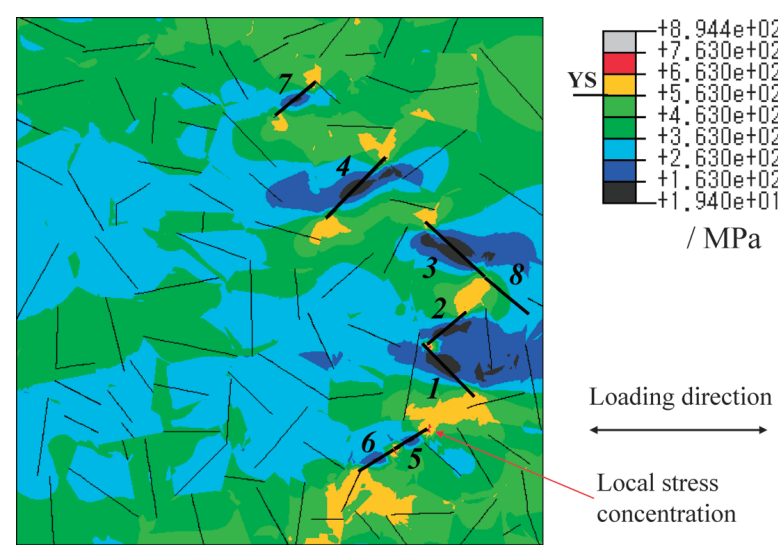

Fig. 8. Mises stress distribution when eight cracks are generated.

difference between the aiming amplitude and the actual amplitude. The stress amplitude decreased as the cycle number increased, which is typically recognized in precipitation strengthened steels, as reported in previous papers. ${ }^{3,7)}$ Klesnil and Lukáśs ${ }^{11)}$ report that whether hardening or softening occurs depends on the yield ratio of the specimen. When the yield ratio is less than 0.71 , fatigue hardening occurs. When that is more than 0.83 , fatigue softening occurs. The yield ratio of the specimen in this study is 0.87 . Consequently, a fatigue softening took place. When $\Delta \varepsilon / 2=$ $0.21 \%$, the stress amplitude monotonously decreases. When $\Delta \varepsilon / 2=0.23 \%$, it reaches a plateau that exceeds about 2000 cycles, which is about $2 \%$ of its fatigue life. When $\Delta \varepsilon / 2=0.25 \%$ and $0.27 \%$, it increases again, after reaching a minimum value. The minimum stress amplitude was seen 
at $1 \%$ and $0.2 \%$ of its fatigue life, respectively. Figure 11 shows the difference between compressive and tensile stress amplitude. Compressive stress was larger than tensile stress. The difference reaches a maximum value in the early stage of the test.

Figure 12 shows the strain-stress hysteresis of the specimens. As the cycle number increases, the width of the hysteresis loop increases. This corresponds to an increase in the plastic strain ratio from fatigue softening. The width of the hysteresis loop for $\Delta \varepsilon / 2=0.27 \%$ is larger than that for

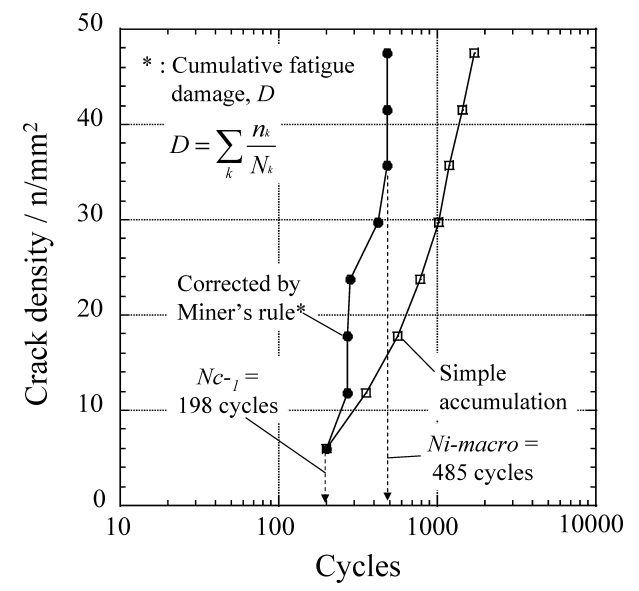

Fig. 9. Relationship between crack density and cycle number.

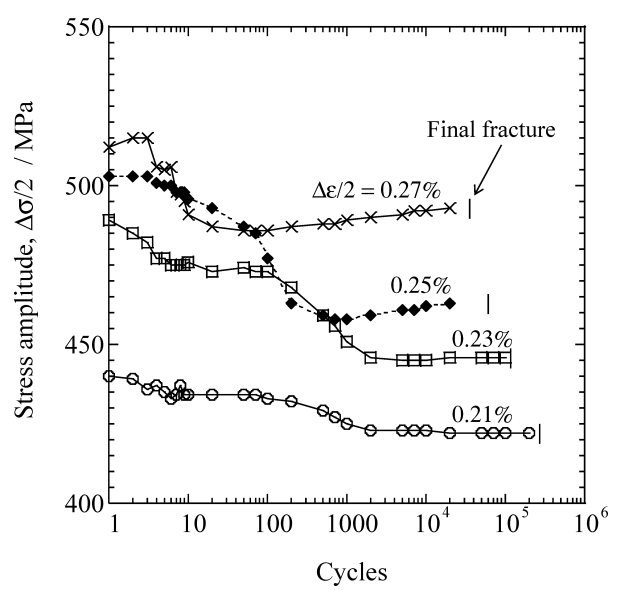

Fig. 10. Relationship between stress amplitude and cycle number.
$\Delta \varepsilon / 2=0.23 \%$. Figure 13 shows the SEM micrographs of the fractured surface. The cracks initiated at the surface near the corner and propagated concentrically. The fatigue crack radius was about $2 \mathrm{~mm}$ regardless of the strain amplitude level. Subsidiary cracks were rarely detected by crosssectional observation with SEM. This is consistent with the fact that macroscopic crack initiation happens at a later stage in the fatigue life in this study as shown in Fig. 10

Figure 14 shows TEM micrographs of dislocation structures after 0, 1000 , and 61000 cycles. Distinct dislocation cell structure was not observed even after 61000 cycles in a fractured sample. Only irregular dislocation tangles between the precipitation particles are observed (Fig. 14(d)). These are a typical salient feature of precipitation hardened steels, as reported. . $^{3,11)}$

\section{Discussion}

\subsection{Estimation of Macroscopic Crack Initiation Cycle Number}

Figure 15 shows the experimentally obtained relationship between strain amplitude and fatigue life, $N_{\text {tot }}$. The macroscopic crack initiation cycle number, $\mathrm{Ni}$-macro was remotely estimated from the crack propagation cycle number, $N c$ by the following equation. This is possible because rapid stress decrease that denotes macroscopic cracking

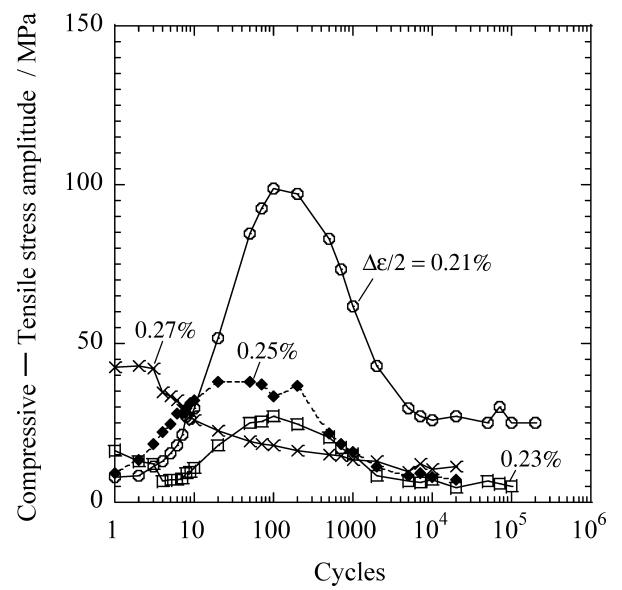

Fig. 11. Difference between compressive and tensile stress amplitude.

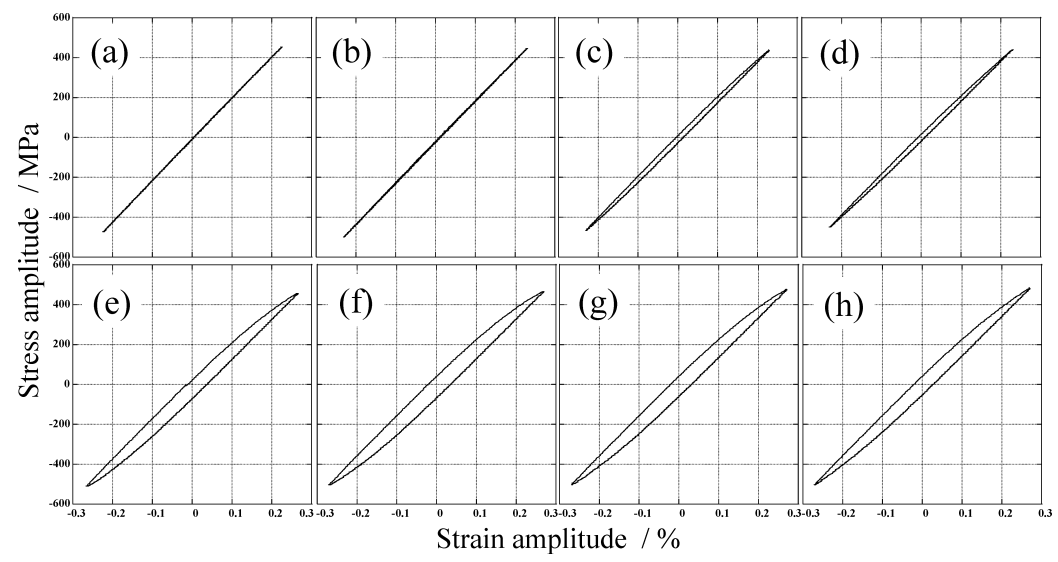

Fig. 12. Strain-stress hysteresis of the specimens: (a) $\Delta \varepsilon / 2=0.23 \%, 10$ cycles, (b) $\Delta \varepsilon / 2=0.23 \%, 100$ cycles, (c) $\Delta \varepsilon / 2=0.23 \%, 1000$ cycles, (d) $\Delta \varepsilon / 2=0.23 \%, 10^{5}$ cycles, (e) $\Delta \varepsilon / 2=0.27 \%, 10$ cycles, (f) $\Delta \varepsilon / 2=0.27 \%, 100$ cycles, (g) $\Delta \varepsilon / 2=0.27 \%, 1000$ cycles, (h) $\Delta \varepsilon / 2=0.27 \%, 10^{4}$ cycles. 
(a)

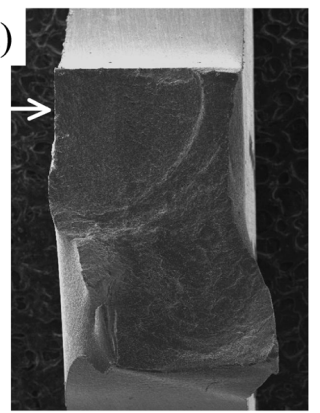

(c)

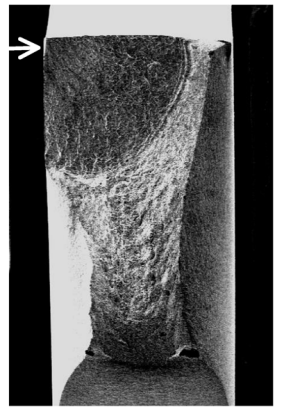

(b)

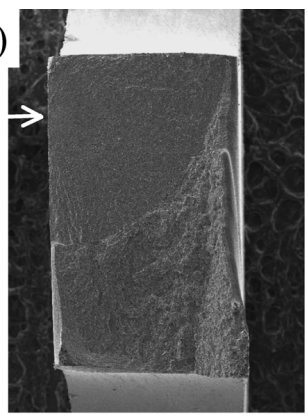

(d)

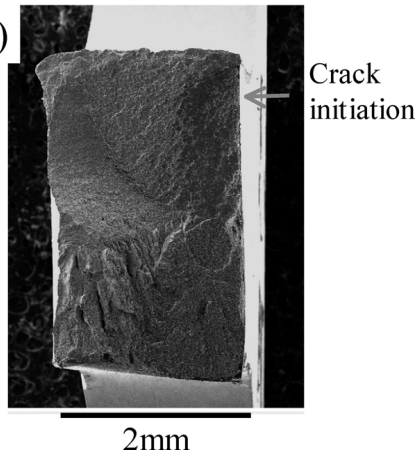

Fig. 13. SEM micrographs of the fractured surface: (a) $\Delta \varepsilon / 2=0.21 \%, \quad 274600 \quad$ cycles, (b) $\Delta \varepsilon / 2=0.23 \%$, 117000 cycles, (c) $\Delta \varepsilon / 2=0.25 \%, 61000$ cycles, (d) $\Delta \varepsilon / 2=0.27 \%, 36000$ cycles.
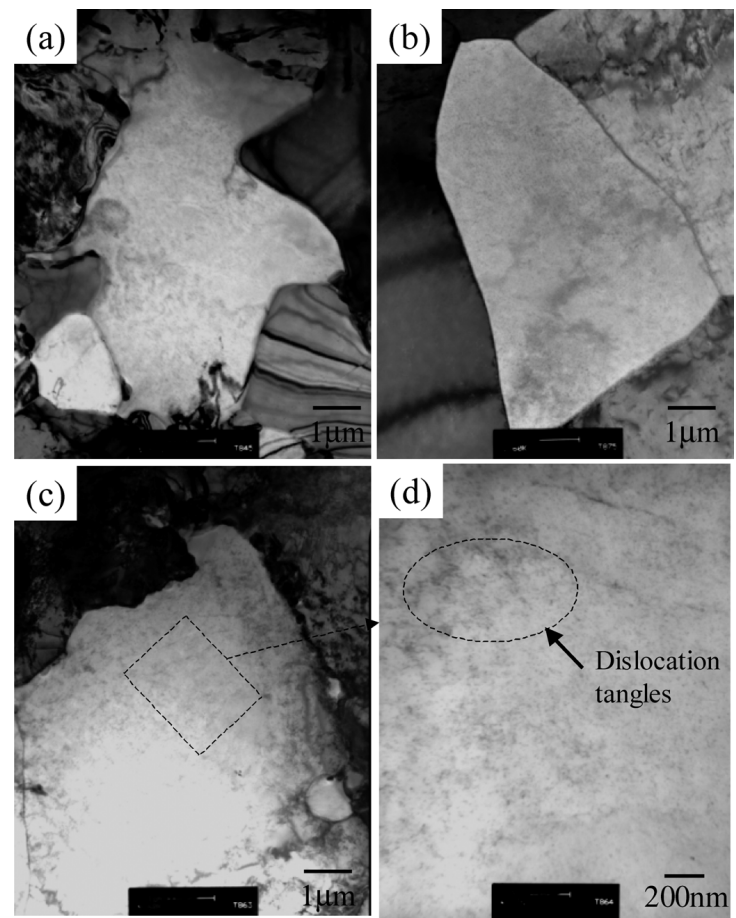

(d)

Fig. 14. TEM micrographs of dislocation structures after (a) 0 , (b) 1000 , (c) 61000 , and (d) 61000 cycles.

was not observed through the intermittent record of strainstress hysteresis curves.

$$
N i-\text { macro }=N_{\text {tot }}-N c \text {. }
$$

$N c$ was calculated by integration of the Paris law as follows. ${ }^{16)}$

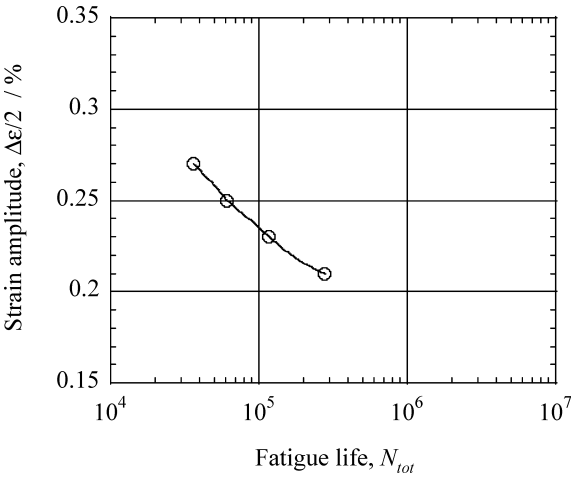

Fig. 15. Experimentally obtained relationship between strain amplitude and fatigue life, $N_{\text {tot }}$.

Table 1. Calculated crack propagation cycle number, $N c$.

(cycles)

\begin{tabular}{c|c|c|c}
\hline$\Delta \varepsilon / 2(\%)$ & $N_{\text {tot }}$ & $N c$ & Ni-macro \\
\hline 0.21 & 274,600 & 6,200 & 268,400 \\
\hline 0.23 & 117,000 & 4,200 & 112,800 \\
\hline 0.25 & 61,000 & 2,900 & 58,100 \\
\hline 0.266 & 40,000 & 2,300 & 37,700 \\
\hline 0.27 & 36,000 & 2,100 & 33,900
\end{tabular}

$$
\begin{aligned}
& N c=\int_{0}^{N c} d N=\int_{a_{i}}^{a_{c}} \frac{1}{C(\Delta K)^{m}} d a=\int_{a_{i}}^{a_{c}} \frac{d a}{C(\Delta \sigma \sqrt{\pi a} \cdot F)^{m}} \\
& =\frac{1}{C(\Delta \sigma \sqrt{\pi} \cdot F)^{m}} \int_{a_{i}}^{a_{c}} \frac{1}{(\sqrt{a})^{m}} d a \\
& =\frac{2\left\{a_{i}^{-(m-2) / 2}-a_{c}^{-(m-2) / 2}\right\}}{(m-2) C(\Delta \sigma \sqrt{\pi} \cdot F)^{m}}
\end{aligned}
$$

where $a_{i}$ and $a_{c}$ are the initial and final fatigue crack lengths. $\Delta \sigma$ and $F$ are the nominal stress and the correction factor, respectively. $C$ and $m$ are material constants. In this study, $a_{i}=16.8 \mu \mathrm{m}$, which is the double the average grain size. $a_{c}=2 \mathrm{~mm}$, which is the thickness of the test specimen in reference to the fracture surface in Fig. 13. According to Eq. $(6),{ }^{17)}$ the value of $F$ is 4.5916 when $\rho=\infty$, which is corresponding to a crack initiated from smooth surface.

$$
F=4.5916 \exp \{-0.6759 \times(s / \rho)\}
$$

where $s$ and $\rho$ are the crack length and the notch root radius, respectively. Furthermore, $F$ was multiplied by the crack correction factor for a surface semicircle $(0.728){ }^{18)}$ The material parameters of Eq. (4) are $C=9.30 \times 10^{-14}$ and $m=4.27$, which are the reported values of $\mathrm{Hv}=235$ carbon steel. ${ }^{16)}$

Calculated $N c$ are listed in Table 1. $N c$ varies from 2100 to 6200 accompanying the change of $\Delta \varepsilon / 2$ from 0.27 to $0.21 \%$. These values are consistent with the fact that rapid stress decrease was not observed until over $80 \%$ of total fatigue life had passed as shown Fig. 10. The fatigue life corresponding to $\Delta \varepsilon / 2=0.266 \%$, which is the assumed value for the simulation, is obtained by interpolation as 40000 cycles. Consequently, $N c$ and Ni-macro are 2300 and 37700 cycles, respectively. 
Table 2. Calculated macroscopic crack initiation cycle number, $\mathrm{Ni}$-macro and $\mathrm{Ni}$ of the first cracking grain, $\mathrm{Ni} i_{-1}$.

\begin{tabular}{c|c|c}
\hline & Simulation & Experimental \\
\hline$d_{f}(\mu \mathrm{m})$ & 52 & 8.4 \\
\hline$N i{ }_{I}$ (cycle) & 198 & 15,000 \\
\hline $\mathrm{Ni-macro}$ (cycle) & 498 & 37,700 \\
\hline
\end{tabular}

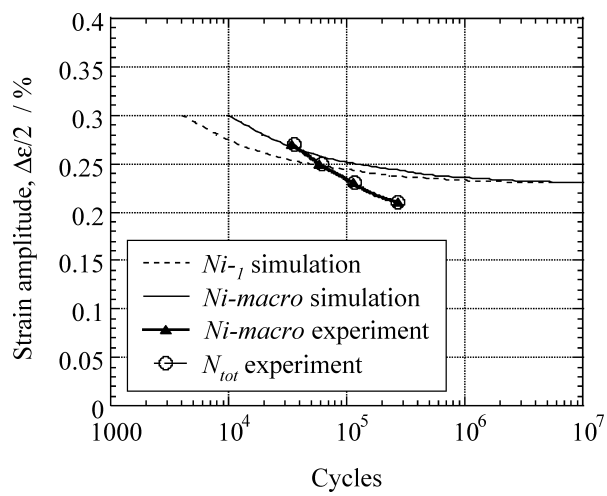

Fig. 16. Relationship between strain amplitude and fatigue-life of the experiment and simulation.

\subsection{Back-calculation of the $\boldsymbol{k}$ Value}

By comparing the analytical model and the actual fatigue test, the $k$ value for the steel used in this study was back calculated. $\mathrm{Ni}$-macro and $\mathrm{Ni}$ of the first cracking grain, $\mathrm{Ni} i_{-1}$ are listed in Table 2. $N i_{-1}$ for the experimental data was calculated on a pro-rate basis. The numerically simulated data of the first cracking grain, $\Delta \tau_{1}=212 \mathrm{MPa}, d_{1}=1.31 d_{f}$, and $N i$-macro $=37700$, were substituted into Eq. (1). Then, the $k$ value was calculated to be $183 \mathrm{MPa}$ for the steel. The difference between the initially assigned and the back-calculated $k$ values may be due to the difference in the strength levels between S35C and the steel used in this study.

\subsection{Correlation between Numerical Simulation and Experimental Results}

Figure 16 shows the relationship between strain amplitude and fatigue-life in the experiment and the simulation. Equation (1) inherently includes an expression for the fatigue limit when $\Delta \tau \leq 2 k$. The calculated fatigue limit is about $\Delta \varepsilon / 2=0.23 \%$. Meanwhile, the experimental fatigue limit is less than $0.21 \%$. The following are the most probable causes for this difference.

(1) In the calculation, the susceptibility of surface to crack initiation is not considered. In the case of thin-walled steel under plane stress conditions, the surface is usually the crack initiation site.

(2) In the calculation, the specimen is as assumed to be homogeneous. However, the actual specimen has strength inhomogeneity to some extent. Strength variability in the actual specimen may decrease the fatigue strength.

(3) In the calculation, only one slip system is presumed. However, multiple slips may occur in the actual specimen. Multiple slips may moderate the effect of strain amplitude on fatigue life through stress relaxation. This moderation is depicted by alternation of the horizontal and vertical axes in Fig. 16.

(4) Equation (1) assumes that one dislocation piles up per one cycle regardless of the strain amplitude level. However, the dislocation reversibility and the plastic strain ratio may actually change depending on the strain amplitude level and the fatigue stage.

For the sake of further confirmation of the simulation with the experimental results, consideration of above-mentioned factors is needed.

Equation (1) includes a significant grain size effect on the crack initiation cycle. According to the previous studies, ${ }^{1,11,19)}$ the grain size effect on fatigue strength depends on the effect of the yield strength of the steel. In general, the percentage of grain boundary strengthening decreases as the strength level increases, so the effects of grain size on fatigue strength in high tensile strength steel will also have to be discussed.

\section{Conclusions}

Crack initiation in TS $590 \mathrm{MPa}$ grade low-alloy precipitation strengthened steel was numerically simulated based on the Tanaka-Mura model. By taking into account the effects of cyclic strain of the preceding cracks, a definable macroscopic crack initiation cycle was obtained. At the same time, an actual tensile and compression fatigue test was conducted on the same steel. Then, the dependence of fatigue-life on strain amplitude was discussed based on the simulation and experimental results. The conclusions are summarized as follows:

(1) Simulated fatigue cracks tend to be clustered due to local stress concentration induced by preexisting cracks.

(2) When the effects of the cyclic strain of the preceding cracks were taken into account using Miner's rule, the crack density increased more rapidly and an abrupt increase in crack density took place. This definable abrupt increase in crack density can be considered a macroscopic crack initiation in the simulation.

(3) In the experiment, the stress amplitude decreased as the cycle number increased, which is typically recognized in precipitation strengthened steels. Distinct dislocation cell structure was not observed by TEM analysis.

(4) The experimental strain fatigue limit was, to some extent, lower than that in the simulation. Surface effects, specimen homogeneity, slip system selection, and dislocation reversibility were mentioned as probable causes for the difference.

\section{REFERENCES}

1) Y. Mutoh and V. M. Radhakrishnan: Trans. ASME, 108 (1986), 174.

2) T. Magnin, C. Ramade, J. Lepioux and L. P. Kubin: Mater. Sci. Eng., A118 (1989), 41

3) M. Mizui, H. Takechi and T. Sekine: Tetsu-to-Hagané, 76 (1990), 414.

4) T. Kitano, T. Tagawa, S. Aihara, M. D. Chappeti and T. Miyata: Tetsu-to-Hagané, 83 (1997), 401.

5) M. Kurita, M. Yamamoto and K. Kunishige: Tetsu-to-Hagané, 85 (1999), 771.

6) K. S. Chan: Metall. Mater. Trans. A, 34A (2003), 43.

7) T. Yokoi, M. Takahashi, N. Maruyama and M. Sugiyama: Shinnittetsu Giho, 381 (2004), 45.

8) K. Ushioda, S. Goto, Y. Komatsu, A. Hoshino and S. Takebayashi: Tetsu-to-Hagané, 94 (2008), 321.

9) T. B. Hilditch, I. B. Timokhina, L. T. Robertson, E. V. Pereloma and 
ISIJ International, Vol. 50 (2010), No. 11

P. D. Hodgson: Metall. Mater. Trans. A, 40A (2009), 342.

10) C. Luo, J. Wei, M. P. Garcia, A. Chattopadhyay and P. Peralta: AIAA J., 47 (2009), 2567.

11) M. Klesnil and P. Lukáš: Fatigue of Metallic Materials, Second Revised ed., Institute of Phycal Metallurgy of the Czechoslovak Academy of Science, (1992)

12) K. Tanaka and T. Mura: J. Appl. Mech., 48 (1981), 97.

13) A. Brückner-Foit and X. Huang: Int. J. Fatigue, 28 (2006), 963.

14) T. Hoshide and K. Kusuura: Fatigue Fract. Eng. Mater. Struct., 21
(1998), 201.

15) M. A. Miner: J. Appl. Mech., 12 (1945), A159.

16) S. Toyoda, Y. Ishiguro, Y. Kawabata, K. Sakata, A. Sato and J. Sakai: J. Solid Mech. Mater. Eng., 3 (2009), 114.

17) A. P. Kfouri: Ph.D Thesis, University of Cambridge, (1970), 132.

18) K. Tanaka, Y. Akiniwa, K. Morita and M. Wakita: J. Soc. Mater. Sci. Jpn., 57 (2008), 140.

19) K. Tanaka: Meijo Univ. Sci. Eng. Dep. Rep., 48 (2008), 67. 